\title{
Description of the Open Anterograde Anatomic Radical Retropubic Prostatectomy Technique
}

Fabricio BC*, Ronaldo D, Alexandro da Silva E, Figueiredo TF, Celso Lara C, Fernanda P, Emanuel FC and Henrique M

Pedro Ernesto University Hospital, Rio de Janeiro State University, Rio de Janeiro, Brazil

\begin{abstract}
Background: This study aimed to reproduce the anterograde technique used in minimally invasive robotic and laparoscopic radical prostatectomy through an open retropubic approach without increasing procedural complexity or cost. The main surgical steps of this technique follow the recommendations of the 2012 Pasadena Consensus Panel for Robotic Surgery modified by the authors to adapt to open retropubic approach.
\end{abstract}

Methods: Ten consecutive eligible men with localized prostate cancer underwent open anterograde anatomic radical retropubic prostatectomy with urethrovesical anastomosis by running suture, consistent with the Pasadena consensus procedure for robotic radical prostatectomy.

Results: All procedures were completed as planned (mean procedure duration, 145.5 minutes; hospital stay, 3.4 days with 3 days of suction drainage; duration of indwelling vesical catheterization, 8 days). At the 1-year follow-up, all patients achieved oncological control. Eight patients (80\%) achieved urinary continence within 90 days; five $(50 \%)$ were continent immediately after indwelling catheter removal. Four minor complications occurred (Clavien-Dindo grade I and IIla).

Conclusion: Open anterograde anatomic radical retropubic prostatectomy allowed the accomplishment of the same technique used in the minimally invasive surgery through the open approach. However, it is necessary to confirm these results through a study with an adequate number of patients comparing with the gold standard technique.

Keywords: Minimally invasive surgical procedures; Prostatic neoplasms; Prostatectomy; Urologic surgical procedures

Abbreviation: PSA: Prostate-Specific Antigen; PSM: Positive Surgical Margin; TNM: Tumour, Node, Metastasis classification; ICIQSF: International Consultation on Incontinence Questionnaire Short Form

\section{Introduction}

Radical prostatectomy is the treatment of choice for localized prostate cancer in patients with a long life expectancy. The refinement of surgical technique and the incorporation of new technologies such as robotics have contributed to better oncological and functional outcomes, as well as leading to the classification of this procedure as minimally invasive surgery $[1,2]$. The laparoscopic prostatectomy technique was first developed in 1997 by Shuessler et al. [3] and improved over time. The robotic system, considered a major breakthrough in the modern treatment of prostate cancer, was introduced in 2000 [4]. Laparoscopic radical prostatectomy (LRP) and robot-assisted radical prostatectomy (RARP) are currently well-established procedures and have been adopted at major urological centers worldwide. Both methods were discussed in detail by the Pasadena Consensus Panel in 2012 [2]. RARP has become the leading surgical technique in the United States, and its adoption is also expanding in other countries. Several systematic reviews and randomized controlled clinical trials have indicated that RARP and LRP are advantageous for the preservation of continence and recovery of potency, despite the absence of high-quality randomized controlled prospective trials supporting their superiority over open radical retropubic prostatectomy (RRP) and the significant disparity between video-assisted anterograde technique (laparoscopic or robotic) and the open retrograde Walsh RRP $[2,5,6]$. Furthermore, the high cost of robotic surgery has limited its use in developing countries such as Brazil, where the incidence of prostate cancer was estimated at 61,200 new cases in 2016, with an overall risk of 61.82 per 100,000 men $[5,7]$. We wished to investigate whether we could offer this new surgical technique to patients who do not have access to these expensive and complex technologies; thus, the aim of this study was to perform the same operation used in minimally invasive radical prostatectomy through an open retropubic incision without additional cost and with the same materials used in open retrograde prostatectomy. Although open anterograde prostatectomy has been described since the work of Campbell in 1959 [8-10]. The technique proposed in the present article differs from the original anterograde prostatectomy in terms of the anatomical dissection of the bladder neck and seminal vesicles and preservation of the neurovascular bundle with lateral and retroprostatic anterograde dissection to the apex and urethrovesical anastomosis with running suture [2,9-11].

\section{Materials and Methods}

This pilot study included a cohort of 10 consecutive eligible male patients treated at the urology clinic at our institution who underwent unilateral or bilateral nerve-sparing anatomic radical retropubic prostatectomy (Table 1) with urethrovesical anastomosis by running suture [2]. This study was approved by the local Ethics Committee, and informed consent was obtained from all individual participants before any study procedures indicating that they were aware they would be operated on with a different technique than usual (Table 1).

\section{Detail of the surgical technique in ten steps}

Patient positioned in dorsal decubitus with mild Trendelenburg

*Corresponding author: Fabricio BC, Urology Service, Pedro Ernesto University Hospital, Rio de Janeiro State University, Rio de Janeiro 20550-900, Brazil, Tel: +5521999869881; E-mail: carrerette2@gmail.com

Received December 04, 2017; Accepted December 13, 2017; Published December 20, 2017

Citation: Fabricio BC, Ronaldo D, Silva ADE, Figueiredo TF, Lara CC, et al. (2017) Description of the Open Anterograde Anatomic Radical Retropubic Prostatectomy Technique. Surgery Curr Res 7: 304. doi: 10.4172/2161-1076.1000304

Copyright: (c) 2017 Fabricio BC, et al. This is an open-access article distributed under the terms of the Creative Commons Attribution License, which permits unrestricted use, distribution, and reproduction in any medium, provided the original author and source are credited. 
Citation: Fabricio BC, Ronaldo D, Silva ADE, Figueiredo TF, Lara CC, et al. (2017) Description of the Open Anterograde Anatomic Radical Retropubic Prostatectomy Technique. Surgery Curr Res 7: 304. doi: 10.4172/2161-1076.1000304

Page 2 of 6

\begin{tabular}{|c|c|c|c|c|c|c|c|}
\hline \multicolumn{2}{|c|}{ Demographic data } & \multicolumn{6}{|c|}{ Tumor characteristics } \\
\hline \multirow[b]{2}{*}{ Patient number } & \multirow{2}{*}{$\begin{array}{l}\text { Age } \\
\text { years }\end{array}$} & \multirow{2}{*}{$\begin{array}{c}\text { Serum PSA } \\
\text { ng/dL }\end{array}$} & \multirow{2}{*}{$\begin{array}{c}\text { Prostate } \\
\text { volume } \\
\text { cc }^{3}\end{array}$} & \multirow{2}{*}{\multicolumn{2}{|c|}{ Prostate Biopsy }} & \multirow{2}{*}{$\begin{array}{l}\text { D'Amico risk } \\
\text { classification }\end{array}$} & \multirow[b]{2}{*}{ Digital rectal examination } \\
\hline & & & & & & & \\
\hline 1 & 53 & 5.4 & 20 & Bilateral & $7(3+4)$ & Intermediate & Normal \\
\hline 2 & 65 & 2.9 & 40 & Left & $7(4+3)$ & Intermediate & Left palpable nodule \\
\hline 3 & 58 & 10.4 & 30 & Right & $6(3+3)$ & Intermediate & Increased prostate without nodule \\
\hline 4 & 61 & 3.68 & 56 & Left & $7(3+4)$ & Intermediate & Left palpable nodule \\
\hline 5 & 61 & 11.83 & 32 & Bilateral & $6(3+3)$ & Intermediate & Increased prostate without nodule \\
\hline 6 & 69 & 7.5 & 27 & Left & $7(4+3)$ & Intermediate & Left palpable nodule \\
\hline 7 & 57 & 14.53 & 40 & Bilateral & $6(3+3)$ & Intermediate & Increased prostate without nodule \\
\hline 8 & 68 & 3.49 & 50 & Right & $6(3+3)$ & Low & Right palpable nodule \\
\hline 9 & 62 & 4.29 & 40 & Right & * & Low & Increased prostate without nodule \\
\hline 10 & 71 & 23 & 50 & Right & $6(3+3)$ & High & Increased prostate without nodule \\
\hline Mean & 62.5 & 8.69 & 38.5 & $\begin{array}{c}\text { (7) } 70 \% \\
\text { unilateral }\end{array}$ & (5) $50 \%=$ G 6 & $\begin{array}{c}(7) 70 \% \\
\text { intermediate }\end{array}$ & (4) $40 \%$ with palpable nodule \\
\hline Range & $53-71$ & $2.9-23$ & $20-56$ & & & (1) $10 \%$ high & (5) $50 \%$ enlargement without nodule \\
\hline
\end{tabular}

Table 1: Preoperative demographic data and tumor characteristics. Serum PSA, prostate volume, prostate biopsy characteristics, D’Amico risk classification, and digital rectal examination.

and slight surgical table flexion (on the level of the iliac crest), infraumbilical midline incision. Incision of endopelvic fascia and section of puboprostatic ligaments with ligation of the dorsal vascular complex without dividing, using Vicryl suture 2.0 or 0 . The dissection of the prostate begins toward anterograde approach, i.e., from bladder neck to the apex, with careful bladder neck dissection and preservation, when possible, as it is separated from the prostate (Figures 1A and 1B). Dissection of the space posterior to the prostate and bladder neck with identification of ejaculatory ducts and seminal vesicles. Meticulous dissection of these structures with minimal use of cautery and traction. Prostate's lateral vascular pedicule dissection and ligation with absorbable suture, such as vicryl 2.0 or 3.0 (Figure 1C). Meticulous retro prostatic dissection with preservation of the posterior layer of Denonvilliers' fascia (which contains communicating nerve fibers) can be left on the rectum. Liberation of the prostate until its apex dorsaly. Uni or bilateral nerve-sparing when indicated, through a careful lateral dissection of the prostate, without the use of the electro cautery, until its apex. Maximum preservation may be obtained by following the plane between the prostatic capsule and the multilayer tissue of the prostatic fascia when possible (Figure 1D).

Meticulous and careful dissection of the prostatic apex and urethra (Figures $1 \mathrm{E}$ and $1 \mathrm{~F}$ ). Section of the dorsal venous plexus with the traction of the prostate and urethra exposure to be sectioned near the apex, preserving a good extension of the abdominal urethra to facilitate the urethrovesical anastomosis. Exeresis of the surgical specimen (Figures $1 \mathrm{E}$ and $1 \mathrm{~F}$ ). Urethrovesical anastomosis, without bladder neck reconfiguration or eversion of the bladder mucosa. Anastomosis confectioned with two monofilament, absorbable 3.0 sutures united at the end to perform a single running suture as described by Van Velthoven et al. [11]. The first stitch is made through the bladder from the outside to the mucosa and then passed through the urethra from the mucosa to its outer layer. After completion of the anastomosis' dorsal part we insert an $18 \mathrm{Fr}$. foley catheter through the urethra and the smooth passage of the catheter over the anastomosis into the bladder indicates the good quality of the running suture. Anastomosis completed with stitching its full circunference (Figure 2). The main surgical steps of this technique follow the recommendations of the 2012 Pasadena Consensus Panel for Robotic Surgery [2], modified by the authors to adapt to open retro-pubic surgery: anterograde dissection with ligation of the dorsal vascular complex without dividing, preservation of the bladder neck,

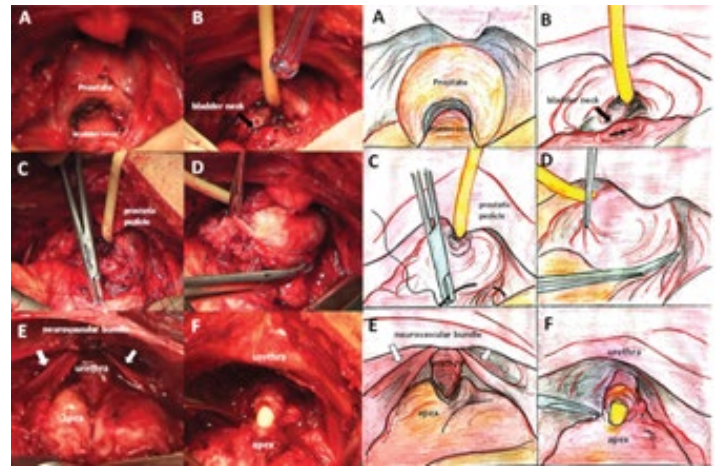

Figure 1: Schematic drawing and surgery photography showing anterograde prostate dissection. A and B) Careful bladder neck dissection and preservation (black arrows). C and D) Prostate's lateral vascular pedicule dissection and ligation, nerve-sparing through a careful lateral dissection of the prostate. $E$ and F) Dissection of the prostatic apex and urethra with observation of the bilateral neurovascular bundle (white arrows).
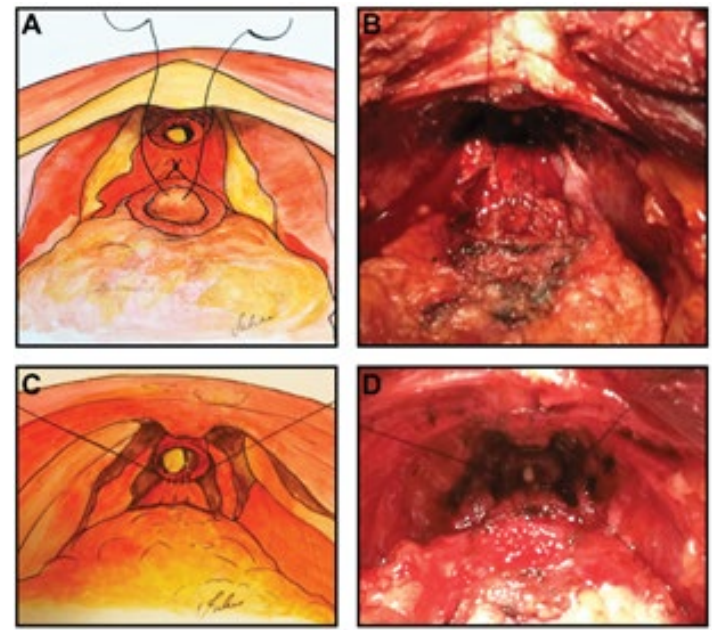

Figure 2: Schematic drawing and surgery photography showing anastomosis confection with two monofilament, absorbable 3-0 sutures joined at their ends to perform a single running suture. The first stitch is made through the bladder from the outside to the mucosa (A and $B)$. Completion of the dorsal half of the anastomosis (C and D). 
nerve sparing, preservation of the posterior layer of Denonvilliers' fascia remaining on the rectum, preservation of the abdominal urethra, and confection of the anastomosis with a running suture (Figures 1 and 2). All patients were operated on by second-year residents of the urological program, aided by a professor from our institution (FBC). Anesthesia for the procedures consisted of spinal block with or without venous sedatives (Figures 1 and 2).

\section{Outcome measures}

Several parameters were assessed to evaluate the feasibility of the proposed new technique. The time of surgery and the ability to complete it successfully using the proposed technique without conversion to retrograde-technique RRP were the main outcomes of this study, along with intraoperative complications, the need for blood transfusion, and postoperative complications such as prolonged drainage, hospital stay, and duration of urethral catheter placement [12]. The secondary outcome measures considered were oncological and functional control. Oncological control was assessed by histopathological analysis of the surgical margins and serum prostate-specific antigen (PSA) measurement to evaluate biochemical recurrence 30 days and 1 year after surgery. Functional control was evaluated by urinary continence. Urine leakage was assessed on the basis of clinical history with a simple question about involuntary urinary loss ("Do you have involuntary loss of urine") and pad use, immediately after removal of the urinary catheter and at 1 month, 3 months, and 1 year postoperatively. Patients were considered continent if they answered that they never leak urine and used no pads or only one security pad per day, which remained dry. Continence was also evaluated using the self-administered International Consultation on Incontinence Questionnaire Short Form (ICIQ-SF), in which question 3 assesses the frequency of incontinence ("How often do you leak urine?") with possible responses of "Never" (score 0), "About once a week or less" (score 1), "Two or three times a week" (score 2), "About once a day" (score 3 ), "Several times a day" (score 4), and "All the time" (score 5). Continence was defined as one or fewer incontinence episodes per week, corresponding to scores 0 or 1 on question 3 of the ICIQ-SF. The extent to which urinary incontinence was bothersome was evaluated by ICIQ-SF question 5 , which asks, "Overall, how much does leaking urine interfere with your everyday life?" Patients selected a number from 0 ("Not at all") to 10 ("A great deal"). All patients were evaluated in postoperative consultations at 7 days for removal of the bladder catheter and skin stitches, and after 1 month, 3 months, and 1 year, as well as at any follow-up, where patients were evaluated and treated for surgical or medical complications. At all visits, complications from surgery were evaluated in addition to serum PSA concentration and continence as described above. Statistical Analysis Categorical variables were expressed as number and percentage. Continuous variables were expressed as mean and range. Due to the small number of patients, statistical comparison between groups was not possible. SPSS PASW (version 22, IBM, Armonk, NY, USA) software was used for all analysis.

\section{Results}

\section{Preoperative characteristics}

Patients' age ranged from 53 to 71 years (mean 62.5 years). All men had elevated PSA and/or abnormal prostatic digital rectal examination and localized prostate cancer diagnosed by prostate biopsy. Using the D'Amico risk classification [13,14]. Seven patients were classified as being at intermediate risk, two at low risk, and one at high risk. Digital rectal examination detected a nodule in four patients and indicated prostate enlargement without palpable nodule in five patients; the findings were normal in one patient. The one patient who had PSA of $23.00 \mathrm{ng} / \mathrm{mL}$ had additional negative screening for metastasis with computed tomography (CT) and bone scintigraphy (Table 1). Immunohistochemistry without Gleason classification. Abbreviation: PSA: prostate-specific antigen.

\section{Postoperative characteristics}

All procedures were completed as planned, without conversion to retrograde Walsh RRP and with preservation of the bladder neck and running suture anastomosis. Pelvic bilateral lymphadenectomy (either conventional or expanded) was performed in all cases but one (defined as low risk by the D'Amico classification). Bilateral nerve sparing was performed in seven patients, and unilateral nerve sparing in three patients. Surgery had a mean duration of 145.5 minutes (range 90-200), and mean hospital stay was 3.4 days (range $3-5$ ), with a mean of 3.1 days (range 3-4) with suction drainage. The mean duration of indwelling vesical catheterization was 8 days (range 7-16) (Table 2). One perioperative complication occurred: anterior bladder wall injury next to the bladder neck caused by the traction forceps. The injury was identified and corrected immediately. During postoperative follow-up, four complications were noted, classified by the Clavien-Dindo system as grade I and IIIa [12]. The patient who experienced the perioperative bladder injury had two complications grade I, demonstrated larger drainage volume, and we detected $276 \mathrm{ml}$ of pelvic fluid collection by ultrasound on postoperative day 7 (grade I). The vesical catheter was maintained until postoperative day 16 , with full resolution. The same patient also presented surgical wound infection, which was treated with a conservative approach (grade I). Two months postoperatively, another patient developed an infected lymphocele between the bladder and the abdominal wall due to lymphadenectomy. This patient was completely continent (without pads) and presented hypo-gastric pain, local swelling, and polacyuria. Ultrasound confirmed a hypogastric fluid collection of $500 \mathrm{ml}$, which was drained through open surgery under local anesthesia (grade IIIa). Finally, one patient developed bladder neck sclerosis, sequelae diagnosed one year after surgery, with de novo urge incontinence that required endoscopic treatment. Based on pathology results, Gleason score was upgraded in half of the patients (50\%). Six patients had a surgical Tumour, Node, Metastasis classification (TNM) (14) of pT2c N0, one was classified as pT2a Nx, one as pT3a N0, and one as pT3b N0. One patient had evidence of bilateral lymph node metastasis and was assigned to stage pT3a N1. Surgical margins were positive in two patients with pT2 and one patients with pT3 disease (Table 2). All patients had achieved a serum PSA $\leq 0.05 \mathrm{ng} /$ $\mathrm{dL}$ at 1 year postoperatively, including one patient with pathological staging of pT3 with a positive node (N1) and Gleason score of $7(4+3)$ (Table 2). The assessment of functional control, as indicated by urinary continence, revealed that six patients $(60 \%)$ did not leak urine or use a pad within 16 days after surgery, with five of them (50\%) reporting this outcome immediately after removal of the indwelling catheter. Eight patients ( $80 \%$ ) became continent within 90 days, and nine (90\%) within 1 year. Only one patient remains incontinent, using one pad per day, after 12 months of follow-up (Table 3). a) Overactive bladder syndrome b) bladder neck sclerosis c) security pad dry.

Continence evaluation by clinical history, upon removal of indwelling catheter, at 90 days, and at 1 year. Evaluation of pad use at 24 hours, 30 days, 90 days, and 1 year. Evaluation of ICIQ SF question 1 (incontinence frequency) at 30 days, 90 days, and 1 year. Evaluation of ICIQ SF question 3 (incontinence bothersome) at 30 days, 90 days, and 1 year. Half of the patients (50\%) responded "Not at all" to ICIQ-SF question 5 regarding the burden of incontinence within 30 days after 


\begin{tabular}{|c|c|c|c|c|c|c|c|c|}
\hline \multirow{2}{*}{ Patient number } & \multirow{2}{*}{$\begin{array}{l}\text { Prostate } \\
\text { Volume }\end{array}$} & \multirow{2}{*}{$\begin{array}{l}\text { Duration } \\
\text { of surgery } \\
\text { (min) }\end{array}$} & \multirow{2}{*}{ Hospital stay } & \multirow{2}{*}{ Drainage (days) } & \multirow{2}{*}{$\begin{array}{l}\text { Bladder catheter } \\
\text { (days) }\end{array}$} & \multicolumn{2}{|c|}{ Postoperative serum } & \multirow{2}{*}{ Gleason score } \\
\hline & & & & & & 30 Days & 1 Year & \\
\hline 1 & 23 & 150 & 3 & 3 & 7 & 0 & 0.01 & $7(4+3)$ \\
\hline 2 & 34 & 200 & 3 & 3 & 8 & 0.003 & 0.01 & $7(4+3)$ \\
\hline 3 & 39 & 150 & 4 & 3 & 7 & 0.01 & 0.01 & $7(4+3)$ \\
\hline 4 & 60 & 120 & 3 & 3 & 7 & 0.41 & 0.01 & $7(3+4)$ \\
\hline 5 & 22 & 150 & 5 & 3 & 16 & 0.01 & 0.05 & $7(4+3)$ \\
\hline 6 & 40 & 140 & 4 & 4 & 7 & 0.02 & 0.025 & $7(4+3)$ \\
\hline 7 & 42 & 200 & 3 & 3 & 7 & 0.08 & 0.01 & $7(4+3)$ \\
\hline 8 & 67 & 90 & 3 & 3 & 7 & 0.04 & 0.01 & $6(3+3)$ \\
\hline 9 & 43 & 125 & 3 & 3 & 7 & 0.01 & 0.01 & $6(3+3)$ \\
\hline 10 & 41 & 130 & 3 & 3 & 7 & 0.01 & 0.01 & $7(3+4)$ \\
\hline Mean & 41.1 & 145.5 & 3.4 & 3.1 & 8 & 0.059 & 0.015 & (8) $80 \%=G 7$ \\
\hline Range & $22-67$ & $90-200$ & 03-May & 03-Apr & Jul-16 & $0.0-0.41$ & $0.01-0.05$ & (2) $20 \%=G 6$ \\
\hline
\end{tabular}

Table 2: Postoperative prostate volume, duration of surgery, days of hospitalization, days of drainage, days of bladder catheterization, oncological control with serum PSA Gleason score, TNM stage, and surgical margin.

\begin{tabular}{|c|c|c|c|c|c|c|c|c|}
\hline \multirow{3}{*}{$\begin{array}{l}\text { Patient } \\
\text { number }\end{array}$} & \multicolumn{3}{|c|}{ Continence } & \multirow{2}{*}{\multicolumn{3}{|c|}{ Pad use (number/24 h) }} & \multirow{2}{*}{\multicolumn{2}{|c|}{$\begin{array}{c}\text { ICIQ-SF } \\
\text { question } 3\end{array}$}} \\
\hline & \multicolumn{3}{|c|}{ By clinical history } & & & & & \\
\hline & $\begin{array}{c}\leq 16 \text { days after } \\
\text { surgery }\end{array}$ & 90 days & 1 year & 30 days & 90 days & 1 year & 30 days & 90 days \\
\hline $1 \mathrm{a}$ & incontinent & incontinent & incontinent & 3 & 3 & 1 & 6 & 4 \\
\hline 2 & incontinent & continent & continent & 0 & 0 & 0 & 0 & 0 \\
\hline 3 & continent & continent & continent & 0 & 0 & 0 & 0 & 0 \\
\hline 4 & continent & continent & continent & 0 & 0 & 0 & 2 & 3 \\
\hline 5 & continent & continent & continent & 0 & 0 & 0 & 1 & 1 \\
\hline 6 & continent & continent & continent & 0 & 0 & 0 & 3 & 1 \\
\hline 7 & continent & continent & continent & 0 & 0 & 0 & 0 & 0 \\
\hline $8 a b$ & incontinent & incontinent & continent & 3 & 2 & $1 \mathrm{c}$ & 4 & 4 \\
\hline 9 & continent & continent & continent & 0 & 0 & 0 & 0 & 0 \\
\hline 10 & incontinent & continent & continent & 1 & 0 & 0 & 4 & 3 \\
\hline Continent & $6(60 \%)$ & $8(80 \%)$ & $9(90 \%)$ & $7(70 \%)$ & $8(80 \%)$ & $9(90 \%)$ & $5(50 \%)$ & $6(60 \%)$ \\
\hline Incontinent & $4(40 \%)$ & $2(20 \%)$ & $1(10 \%)$ & $3(30 \%)$ & $2(20 \%)$ & $1(10 \%)$ & $5(50 \%)$ & $4(40 \%)$ \\
\hline
\end{tabular}

Table 3: Functional control with assessment of urinary continence.

surgery, and $80 \%$ responded "Not at all" within 3 months. At 1 year, only the incontinent patient had a score of 6 for ICIQ-SF question 5; all other patients' scores were 0 (Table 3 ).

\section{Discussion}

Open radical retropubic prostatectomy through an anterograde dissection with non-opening of the endopelvic fascia, preservation of the bladder neck, nerve sparing, preservation of the posterior layer of Denonvilliers' fascia remaining on the rectum, preservation of the abdominal urethra, and confection of the anastomosis with a more impermeable running suture may allow for the execution of a more anatomically precise and less invasive operation, independent of the access route for surgery. The development and improvement of surgical technique achieved by LRP and RARP may not be entirely due to their technological advances. The enhanced vision associated with a convenient sitting position, wider operative field, and ability for more precise movements are advantages of robotic-assisted surgery, and are part of evolution in surgical techniques toward better visualization of pelvic anatomical structures such as the cavernous nerve fibers within the periprostatic tissue, seminal vesicles, prostatic pedicle, apex, and urethra [1,2,5,15-18]. This advancement has allowed for the development of a new method to perform an anterograde anatomical dissection technique that is more precise and less invasive than the classical open RRP described in 1983 by Walsh et al. [2,5,6,9$11,15,17,18]$. Our study demonstrates the feasibility of performing this surgery using an open technique, without adding the high costs of new technology. All open anterograde operations were executed with the same technique used in video-assisted surgery (LRP or RARP), following the recommendations of the Pasadena Consensus Panel [2] which were reproduced through the conventional open retro-pubic approach. Although several articles have been published on the topic of anterograde radical prostatectomy, no effort has been directed to describing the entire procedure as in the present report, following the robotic technique described by the 2012 Pasadena consensus step-bystep [2,9-11]. This technique has been previously reported only in video-assisted (laparoscopic or robotic) surgery. The operations were executed by second-year residents of our hospital's urology program, aided by one of the authors, who is a senior surgeon. All surgeries were performed as planned, without conversion to the Walsh technique, and had a similar duration to other RRP techniques $[5,16]$. Therefore, we believe that this surgical technique is both feasible and reproducible, since residents could complete it even in their first procedures supervised by a professor with an average surgical time of 145.5 minutes. This result is comparable to other series reporting mean procedure times of 181 minutes for RRP, 219 minutes for LRP, and 
179.5 minutes for RARP [16]. The ease of the procedure reported here is an advantage compared to video-assisted surgery, for which surgeons typically require more than 200 procedures to reach a plateau in the learning curve [16]. The procedure is initiated with ligation of the dorsal vascular complex without dividing it, with no incision of the endopelvic fascia or puboprostatic ligaments. Preserving all these structures close to the urethral sphincter aids in nerve preservation in addition to reducing bleeding, since much of the blood lost during surgery comes from the plexus of the dorsal vein, the spongy body of the urethra, and the endopelvic fascia. We did not observe significant bleeding necessitating blood transfusion or any perioperative complication related to surgical technique. The bladder injury was caused by traction with forceps used for all open prostatectomies. Initiating prostate dissection through the bladder neck (anterograde approach) facilitates the preservation of the bladder neck and the nerve bundle as well as Denonvilliers' fascia, making it easier to leave the fascia attached to the rectum and avoiding possible injury to several nerve fibers that travel along this path. This technique also facilitates dissection of the prostate apex by helping to preserve the nerve bundle passing next to the urethra and allowing preservation of a greater extent of the urethra (Figure 1). The preservation of the bladder neck together with a greater extension of the abdominal urethra facilitates the more hermetic Van Velthoven running suture anastomosis technique (Figure 2). Therefore, the drainage volume was low, similar to the good results observed for length of hospital stay (mean of 3.4 days, range 3-5 days depending primarily on suction drain volume) and bladder catheterization. These improvements in recovery-related outcomes are benefits of the anterograde technique that have been previously reported for video-assisted surgery; however, the present study is the first to report consistent findings using the same surgical procedure with an open technique $[11,13,18-21]$. In this series, the catheter was typically removed 1 week after surgery. Our mean catheterization time was 8 days, which included the patient who remained catheterized for 16 days. This timeline is already employed in many centers that perform LRP and RARP, which have a mean catheterization time of 5-7 days $[15,16]$. However, at our institution, patients who undergo open retrograde RRP remain catheterized for an average of 2 weeks. In this study, we observed two cases of positive surgical margin in pT2 cancers and one in pT3. Evidence suggests that positive surgical margins in pT2 prostate cancer are potentially avoidable, whereas in pathologic stage pT3 cancers, positive surgical margins are much more frequently associated with the extent of disease. Positive surgical margins therefore should not necessarily be considered to reflect surgical failure [3,22]. In an extensive literature review, rates of positive surgical margin reached $50 \%$ in $\mathrm{pT} 3$ [23]. Another related factor is the number of procedures, with less than 50 cases the positive margin occurred in $36.1 \%$ in pT2 [24]. Oncological control, as indicated by serum PSA $\leq 0.05$, was achieved in all patients within 1 year after surgery, including the patient with advanced stage disease, with tumor extension outside the prostate and lymph node involvement (pT3 N1), which demonstrates the ability of the method to address extra prostatic involvement. Urinary continence is a major marker of surgical success and one of the most important factors affecting patient quality of life, even more so than erectile function [2]. We observed a high rate of early continence in this study immediately after removal of the indwelling catheter in five patients (50\%) and after the use of a pad for only 3 days in another patient. Eight patients (80\%) were continent within 90 days postoperatively, and nine $(90 \%)$ were continent within 1 year, a continence rate rarely encountered among our patients who undergo retrograde RRP. Two patients had urge incontinence for overactive bladder, one due diabetic and stroke in medical history, before surgery and the other compounded by bladder neck sclerosis, this last became continent after endoscopic correction. One patient who was continent immediately after removal of the indwelling catheter began to experience sporadic stress incontinence approximately 90 days postoperatively; at the 1-year follow-up, he was continent by two criteria (clinical history and pad use) and he continued to experience sporadic incontinence upon high physical exertion in his work as a manual laborer (Table 3). Two systematic reviews have demonstrated better continence outcomes among patients who undergo to RARP in comparison with RRP. Ficarra et al. reported a $97 \%$ continence rate at the 1-year follow-up in the RARP group compared to $88 \%$ in the RRP group, with continence defined as urine loss less than once a week. This study also observed earlier continence in the RARP group (25 days vs. 75 days in the RRP group) [17]. Several studies have attempted to develop better methods to preserve continence after radical prostatectomy, although there are no known factors capable of improving this outcome in RRP [6,18]. Postoperative continence appears to be multifactorial. However, anterograde dissection with bladder neck preservation, nerve sparing, non-opening of the endopelvic fascia, preservation of the abdominal urethra, and confection of the anastomosis with a more impermeable running suture (Figure 1) may allow for a more anatomically precise operation that safeguards the structures important for attaining precocious continence after radical prostatectomy. Our finding of earlier continence is consistent with published results for minimally invasive video-assisted procedures (Table 3 ). In conjunction with the recovery of urinary continence, at 30 days after surgery, half of the patients did not find their urinary state bothersome. This number increased to 8 patients within 3 months postoperatively, and at 1 year, only the patient with ongoing incontinence had a score of 6 for ICIQ-SF question 5 (Table 3 ). We did not evaluate erectile function, because it was not assessed in patients prior to diagnosis. At 1 year postoperatively, only one patient experienced erections that allowed them to have sex. This variable is currently being evaluated in a prospective trial.

\section{Conclusion}

We were able to perform open anterograde anatomic radical retropubic prostatectomy with a similar technique to that used in minimally invasive radical prostatectomy, allowing men who do not have access to this new technology to be operated on with the same technique used in robotic or laparoscopic surgery. Furthermore, the open anterograde anatomic radical retropubic prostatectomy method was reproducible by low-volume prostate cancer surgeons; therefore, it may help inexperienced surgeons to develop skills valuable to future training with robotic or laparoscopic techniques. However, this technique should be applied with major limitation while awaiting the outcome of the definitive study that supports the preliminary results.

\section{Study Limitations}

This study has many limitations because it is a pilot study with a small number of patients treated with this technique and the absence of a control group. However, the results of this pilot study have led us to establish a prospective, controlled, randomized clinical trial with a large number of patients to confirm that this technique is not inferior to the classical RRP retrograde technique (ClinicalTrials.gov identifier: NCT02687308)

\section{References}

1. Cathelineau X, Sanchez-Salas R, Barret E, Rozet F, Galiano M, et al. (2010) Radical prostatectomy: evolution of surgical technique from the laparoscopic point of view. Int Braz J Urol 36: 129-340. 
Citation: Fabricio BC, Ronaldo D, Silva ADE, Figueiredo TF, Lara CC, et al. (2017) Description of the Open Anterograde Anatomic Radical Retropubic Prostatectomy Technique. Surgery Curr Res 7: 304. doi: 10.4172/2161-1076.1000304

2. Montorsi F, Wilson TG, Rosen RC, Ahlering TE, Artibani W, et al. (2012) Best practices in robot-assisted radical prostatectomy: recommendations of the Pasadena Consensus Panel. Eur Urol 62: 368-381.

3. Schuessler WW, Schulam PG, Clayman RV, Kavoussi LR (1997) Laparoscopic radical prostatectomy: initial short-term experience. Urology 50: 854-857.

4. Abbou CC, Hoznek A, Salomon L, Lobontiu A, Saint F, et al. (2000) Remote laparoscopic radical prostatectomy carried out with a robot. Report of a case. Prog Urol 10: 520-523

5. Lowrance WT, Tarin TV, Shariat SF (2010) Evidence-based comparison of robotic and open radical prostatectomy. Scientific World Journal 10: 2228-2237.

6. Walsh PC, Lepor H, Eggleston JC (1983) Radical prostatectomy with preservation of sexual function: anatomical and pathological considerations. Prostate 4: 473-485.

7. Schilithz AOC, Lima FCS, Andrade JHV, Oliveira JFP, Santos MO, et al. (2016) Estimativa: incidência de câncer no Brasil / Instituto Nacional de Cânce José Alencar Gomes da Silva - INCA. Fox Print, Rio de Janeiro. Article in Portuguese.

8. Campbell EW (1959) Total prostatectomy with preliminary ligation of the vascular pedicles. J Urol 81: 464-467.

9. Sciarra A, Gentile V, De Matteis A, Dattilo C, Autran Gomez AM, et al. (2008) Long-term experience with an anatomical anterograde approach to radical prostatectomy: Results in terms of positive margin rate. Urol Int 80: 151-156.

10. Sciarra A, Cristini C, Von Heland M, Salciccia S, Gentile V (2010) Randomized trial comparing an anterograde versus a retrograde approach to open radical prostatectomy: Results in terms of positive margin rate. Can Urol Assoc $\mathrm{J} 4$ : 192-198.

11. Van Velthoven RF, Ahlering TE, Peltier A, Skarecky DW, Clayman RV (2003) Technique for laparoscopic running urethrovesical anastomosis: the single knot method. Urology 61: 699-702.

12. Mitropoulos D, Artibani W, Graefen M, Remzi M, Rouprêt M, et al. (2015) Guidelines on reporting and grading of complications after urologic surgical procedures. European Association of Urology.

13. D'Amico AV, Desjardin A, Chung A, Chen MH, Schultz D, et al. (1998) Assessment of outcome prediction models for patients with localized prostate carcinoma managed with radical prostatectomy or external beam radiation therapy. Cancer 82: 1887-1896.
14. Sobin LH, Gospodarowicz M, Wittekind C (2009) TNM classification of malignant tumors (7th edn.). UICC International Union Against Cancer.

15. Ficarra V, Novara G, Fracalanza S, D'Elia C, Secco S, et al. (2009) A prospective, non-randomized trial comparing robot-assisted laparoscopic and retropubic radical prostatectomy in one European institution. BJU Int 104: 534539.

16. Frota R, Turna B, Barros R, Gill IS (2008) Comparison of radical prostatectomy techniques: open, laparoscopic and robotic assisted. Int Braz J Urol 34: 259269.

17. Tewari A, Takenaka A, Mtui E, Horninger W, Peschel R, et al. (2006) The proximal neurovascular plate and the tri-zonal neural architecture around the prostate gland: importance in the athermal robotic technique of nerve-sparing prostatectomy. BJU Int 98: 314-323.

18. Brunocilla E, Pultrone C, Pernetti R, Schiavina R, Martorana G (2012) Preservation of the smooth muscular internal (vesical) sphincter and of the proximal urethra during retropubic radical prostatectomy: description of the technique. Int J Urol 19: 783-785.

19. Ozu C, Hagiuda J, Nakagami Y, Hamada R, Horiguchi Y, et al. (2009) Radical retropubic prostatectomy with running vesicourethral anastomosis and early catheter removal: Our experience. Int J Urol 16: 487-492.

20. Lim JH, Park CM, Kim HK, Park JY (2015) Comparison of perioperative outcomes between running versus interrupted vesicourethral anastomosis in open radical prostatectomy: A single-surgeon experience. Korean J Urol 56 : 443-448.

21. Matsuyama H, Matsumoto H, Nagao K, Harada N, Hara T, et al. (2015) Running suture versus interrupted suture for vesicourethral anastomosis in retropubic radical prostatectomy: A randomized study. Int J Urol 22: 271-277.

22. Yossepowitch O, Bjartell A, Eastham JA, Graefen M, Guillonneau BD, et al (2009) Positive surgical margins in radical prostatectomy: outlining the problem and its long-term consequences. Eur Urol 55: 87-99.

23. Novara G, Ficarra V, Mocellin S, Ahlering TE, Carroll PR, et al. (2012) Systematic review and metaanalysis of studies reporting oncologic outcome after robot-assisted radical prostatectomy. Eur Urol 62: 382-404.

24. Al-Hathal N, El-Hakim A (2013) Perioperative, oncological and functional outcomes of the first robotic prostatectomy program in Quebec: single fellowship-trained surgeon's experience of 250 cases. Can Urol Assoc J 7 : 326-332. 